\title{
FORMAS DE INTERAÇÃO DE TRÊS BAIRROS PERIFÉRICOS COM O PARQUE MUNICIPAL DAS MANGABEIRAS, BELO HORIZONTE, MINAS GERAIS $^{1}$
}

Thaís Helena da Costa Porfírio² ${ }^{2}$ Francisco Candido Cardoso Barreto ${ }^{3}$, Agostinho Lopes de Souza ${ }^{4}$ e Wantuelfer Gonçalves ${ }^{4}$

\begin{abstract}
RESUMO - Este estudo teve como objetivo verificar se as diferenças no padrão socioeconômico dos habitantes de bairros adjacentes ao Parque Municipal das Mangabeiras - PARMA levariam a diferenças na interação dos moradores de cada bairro com essa área. Para tanto, foram analisadas respostas de 30 moradores de cada um dos três bairros imediatamente limítrofes ao PARMA, em Belo Horizonte, capital do Estado de Minas Gerais, os quais responderam a questionários semi-abertos, avaliaram a interação da comunidade com o Parque e com o ambiente e o seu comportamento de conservação. O objetivo geral do estudo foi dar subsídios ao Plano de Educação Ambiental do Parque das Mangabeiras, sendo o específico verificar se as diferenças socioeconômicas influem na interação do morador do entorno com o Parque. Durante as análises dos resultados, observouse que as três comunidades diferiram em relação à freqüência nos programas e projetos do Parque, em relação às desvantagens causadas pela proximidade do PARMA e em relação à importância do Parque, fato corroborado pelas análises estatísticas. No tocante às demais questões, apesar de não terem sido verificadas diferenças estatisticamente significativas, observaram-se diferenças qualitativas nas respostas dadas pelos moradores.
\end{abstract}

Palavras-chave: Unidade de conservação, floresta urbana e desenvolvimento socio-ambiental.

\section{FORMS OF INTERACTION OF THREE OUTLYING AREAS WITH THE MUNICIPAL PARK OF THE MANGABEIRAS, BELO HORIZONTE, MINAS GERAIS}

\begin{abstract}
The present study had as objective to verify if the differences in socioeconomic pattern of the inhabitants of neighborhoods adjacent to the Municipal Park of Mangabeiras - PARMA, would lead to differences in the interaction of inhabitants of each neighborhood with the area. The answers of 30 inhabitants of each one of the three neighborhoods bordering the Mangabeiras Park (PARMA), in Belo Horizonte, capital of the State of Minas Gerais were analyzed. Half-open questionnaires were applied evaluating the interaction of the community with the park and its environment, and the conservation behavior. The general objective of the study was to provide information to the project of Environmental Education of the Mangabeiras Park, being the specific objective to verify if the socioeconomic differences influence in the interaction of the adjacent inhabitant with the Park. The results showed that the three communities differed in relation to the participation in the programs and projects of the Park, in relation to the disadvantages caused by the proximity to the Park and in relation to the importance of its existence, which was corroborated by the statistical analyses. In relation to the other questions, despite the non-occurrence of significant differences, qualitative differences were observed in the answers given by the inhabitants.
\end{abstract}

Keywords: Conservation unit, urban forest, and socioeconomic development.

\footnotetext{
${ }^{1}$ Recebido em $1^{\circ} .09 .05$ e aceito para publicação em 13.09.2006.

2 Programa de Pós-Graduação em Ciência Florestal da UFV. E-mail: <enfthais@ yahoo.com.br>.

${ }^{3}$ Programa de Pós-graduação em Entomologia da UFV. E-mail: <plasmideo@ hotmail.com>.

${ }^{4}$ Departamento de Engenharia Florestal da UFV. E-mail: <alsouza@ufv.br>e <w.goncav@ufv.br>.
} 


\section{INTRODUÇÃO}

De acordo com Miller (1998), floresta urbana pode ser definida como a soma de toda floresta e associação vegetal dentro e no entorno de comunidades densamente povoadas, que geralmente ligam pequenas comunidades rurais às regiões metropolitanas. Uma grande diversidade de vegetação pode ser encontrada nas cidades.

De modo geral, a população urbana não usufrui diretamente dos recursos presentes nas florestas urbanas para sua subsistência. Estas são utilizadas pela população indiretamente, como recreação, desenvolvimento sociocultural, educação ambiental e ecoturismo, entre outros. Contudo, essas florestas sofrem grandes impactos devido à pressão antrópica no seu interior e no seu entorno.

Os habitantes de uma cidade não são uma categoria homogênea e, por isso, têm diferentes necessidades e percepções dos espaços verdes urbanos. De acordo com Chiari e Seedland (2002), as áreas verdes podem apresentar grande papel na integração social quando adequadamente planejadas e usadas. Para tal, o projeto desses espaços têm que considerar as necessidades dos diferentes grupos sociais.

Segundo Metzger (2001), a paisagem é um mosaico heterogêneo formado por unidades interativas, sendo essa heterogeneidade existente para pelo menos um fator, segundo um observador e em determinada escala de observação. Dessa forma, os estudos sobre as florestas urbanas devem levar em consideração a matriz urbana na qual elas estão inseridas.

A partir do pressuposto de que as diferenças socioeconômicas influem na interação dos moradores dos diferentes bairros com o Parque Municipal das Mangabeiras (PARMA), localizado em Belo Horizonte, Minas Gerais, foi realizado o presente estudo, buscando comparar a interação dos moradores dos três bairros periféricos com o parque e seu comportamento de conservação.

\section{MATERIAL E MÉTODOS}

\subsection{Caracterização da Área de Estudo}

De acordo com a PMBH (2003b), o Parque Municipal das Mangabeiras (PARMA), situado no município de Belo Horizonte, Minas Gerais, representa uma área total de 337 ha recobertos por tipologias vegetais representantes do bioma Cerrado, como campos rupestres, matas de galeria e cerrado sensu strito. $\mathrm{O}$ PARMA apresenta grande riqueza hidrológica, abrangendo 14 nascentes.

Em conversas com funcionários do Parque, verificou-se que os principais impactos observados no PARMA estão relacionados ao uso indevido do fogo, captura de animais silvestres, invasão de espécies da fauna doméstica, furtos e outros crimes praticados no interior e entorno da unidade e superpopulação de fauna silvestre devido ao confinamento e à alta oferta de alimentos e poluição dos cursos d'água, entre outras. Tais ameaças são causadas, principalmente, pelos visitantes e pela população do entorno.

Segundo a Prefeitura Municipal de Belo Horizonte (2003a), o entorno do PARMA, aqui considerado como os bairros imediatamente limítrofes à unidade, apresenta uma população próxima de 53.055 habitantes, dos quais $10 \%$ se encontram no Bairro Mangabeiras, e os demais estão proporcionalmente distribuídos entre os Bairros Serra e Aglomerado da Serra. O Bairro Mangabeiras, localizado na face sul do PARMA, caracteriza-se por uma população de classe alta, sendo o Rendimento Nominal Mensal Médio das Pessoas Responsáveis pelos Domicílios Particulares Permanentes (RMM), de 19 salários mínimos. Situado na face oeste do PARMA, o Serra apresenta população de classe social médiaalta, cujo RMM é de 15,5 salários mínimos. No limite noroeste do Parque, situa-se o Aglomerado da Serra, que é um complexo de vilas urbanizadas, sendo uma das primeiras áreas de Belo Horizonte a ser invadida por pessoas desabrigadas e, posteriormente, tornarse uma favela. Nos dias de hoje, o Aglomerado da Serra apresenta rede de saneamento básico, distribuição de luz e áreas públicas pavimentadas. A renda média mensal por chefe de família (RMM) nesSa Comunidade é de 1,4 salário mínimo, correspondendo a 7,25\% do mesmo parâmetro para o Bairro Mangabeiras.

\subsection{Aquisição dos dados}

Foi usado um questionário contendo 13 perguntas a respeito de dados pessoais dos moradores dos Bairros Serra, Mangabeiras e Aglomerado da Serra, bem como de sua interação com o PARMA. Para tal, baseou-se em outros questionários utilizados em estudos feitos no entorno de unidades de conservação situadas em meio rural, como em Miranda (2001) e Ditt (2002). O 
questionário foi aplicado a 30 moradores de cada um dos bairros, Mangabeiras, Serra e Aglomerado da Serra, totalizando 90 moradores analisados.

O questionário aplicado foi do tipo semi-estruturado, pelo simples fato de suas perguntas permitirem a valoração numérica das respostas atribuídas a elas e também criarem oportunidades para depoimentos dos entrevistados, provocando a emergência de assuntos que passariam desapercebidos caso ele fosse elaborado com questões somente fechadas (DITT, 2002). Os assuntos abordados em cada uma das questões estão descritos no Quadro 1.

Foram realizadas três visitas aos Bairros Mangabeiras e Serra, no intuito de aplicar os questionários aos moradores. No entanto, os moradores acionados nessa ocasião não aceitaram participar da pesquisa. A partir de então, foram procuradas as respectivas Associações Comunitárias, que indicaram o uso do telefone como a melhor forma de contado com moradores, haja vista questões de segurança pública. Tais associações indicaram as pessoas que se disporiam a responder ao questionário.

No Bairro Aglomerado da Serra, também com o auxílio da Associação Comunitária, os questionários foram aplicados pessoalmente, sendo os moradores escolhidos de forma aleatória.

Para a análise dos resultados, foram atribuídos valores às respostas das questões 5 a 12, com o objetivo de quantificar a afetividade desses moradores pelo Parque e pelo meio ambiente. No Quadro 2, apresentouse o esquema de pontuações das respostas.

Quadro 1 - Categorias e assuntos abordados em cada questão Table 1 - Category and topics includedin each question

\begin{tabular}{ll}
\hline \multicolumn{1}{c}{ Categorias } & \multicolumn{1}{c}{ Assuntos } \\
\hline Características do morador & Sexo, idade, escolaridade, profissão \\
Afetividade com o parque & $\begin{array}{l}\text { Freqüência de visitas e de presença em eventos, } \\
\text { conhecimentos dos recursos, participação }\end{array}$ \\
Afetividade com o meio ambiente & $\begin{array}{l}\text { Motivo de uso do parque, problemas devido } \\
\text { à proximidade com o parque, importância e } \\
\text { visitação em outras áreas naturais }\end{array}$ \\
Informações gerais & Sugestões e críticas \\
\hline
\end{tabular}

Quadro 2 - Valores atribuídos às questões e os respectivos testes estatísticos

Table 2 -Values attributed to the questions and their respective statistic tests

\begin{tabular}{|c|c|c|}
\hline Questão (número e tema) & Pontuações & Teste estatístico \\
\hline 5 (freqüência de visitas ao parque) & $\begin{array}{l}\text { Semanal: } 3 \text { pontos } \\
\text { Mensal: } 2 \text { pontos } \\
\text { Anual: } 1 \text { ponto } \\
\text { Não freqüenta: } 0 \text { ponto }\end{array}$ & Kruskal-Wallis \\
\hline 6 (fatores que motivam a visita) & Pontuação igual ao número de respostas & Kruskal-Wallis \\
\hline 7 (freqüência em eventos) & Pontuação igual ao número de respostas & Kruskal-Wallis \\
\hline $\begin{array}{l}8 \text { (problemas com a proximidade } \\
\text { entre a residência e o parque) }\end{array}$ & $\begin{array}{l}\text { Sim: } 0 \text { ponto } \\
\text { Não: } 1 \text { ponto }\end{array}$ & Qui-quadrado \\
\hline 9 (importância do parque) & $\begin{array}{l}\text { Ambiental e sociocultural: } 3 \text { pontos } \\
\text { Ambiental: } 2 \text { pontos } \\
\text { Sociocultural: } 1 \text { ponto } \\
\text { Outros: } 0 \text { ponto }\end{array}$ & Kruskal-Wallis \\
\hline $\begin{array}{l}10 \text { (número de espécies animais e, } \\
\text { ou, vegetais que conhece) }\end{array}$ & $\begin{array}{l}\text { Pontuação igual ao número de } \\
\text { espécies citadas }\end{array}$ & Kruskal-Wallis \\
\hline 11 (visitação a outras áreas naturais) & $\begin{array}{l}\text { Sim: } 1 \text { ponto } \\
\text { Não: } 0 \text { ponto }\end{array}$ & Qui-quadrado \\
\hline 12 (participação em eventos de gestão) & $\begin{array}{l}\text { Sim: } 1 \text { ponto } \\
\text { Não: } 0 \text { ponto }\end{array}$ & Qui-quadrado \\
\hline
\end{tabular}


As respostas dos moradores foram separadas por bairro, sendo analisadas estatisticamente através dos testes Qui-quadrado ( $\left.\mathrm{X}^{2}\right)$ e Kruskal-Wallis (K-W), dependendo se a questão permitia respostas binárias, ou se era uma questão não-paramétrica, respectivamente.

As questões 5,6,7,9 e 10 foram analisadas mediante o emprego do teste t, de Kruskal-Wallis, pelo fato de as referidas questões apresentarem mais de duas possíveis respostas e, em algumas delas, mais de uma resposta por entrevistado.

O teste Kruskal-Wallis é indicado para situações em que existem mais de dois tratamentos aplicados a grupos diferentes (SAMPAIO, 1998). Nesses casos, Kruskal e Wallis (1952) sugeriram o procedimento descrito a seguir, para $\mathrm{k}$ tratamentos $(\mathrm{k}>2)$ :

1. Ordenar todas as observações da menor para a maior. Havendo valores observados em empate, a ordenação média deverá substituir as ordenações correspondentes.

2. Calcular a soma das ordenações $\left(\mathrm{R}_{\mathrm{i}}\right)$ para cada grupo experimental i.

3. Calcular o valor de T, pela aplicação da fórmula:

$$
T=\frac{1}{S^{2}}\left[\sum_{i=1}^{k} R_{i}^{2}-\frac{N(N+1)^{2}}{4}\right]
$$

em que:

$n_{i j}=$ número de entrevistas por grupo $\left(n_{i j}=30\right)$;

$$
S^{2}=\frac{1}{N-1}\left[\sum X_{i j}^{2}-\frac{N(N+1)^{2}}{4}\right]
$$

$N=$ número total de entrevistas $(N=90)$;

$R j=$ soma das ordenações para cada grupo $i$;

$X_{i j}=$ ordenação de cada observação $i$ de cada bairro $j$;

$i=$ cada um dos entrevistados $(i=1,2, \ldots, 30) ; \mathrm{e}$

$j=$ cada um dos bairros $(j=1,2,3)$.

No presente trabalho, considerou-se como tratamentos cada um dos bairros, sendo que $j$ variou de 1 a 3 , referindo-se a cada um dos bairros; $i$ variou de 1 a 30, referindo-se a cada uma das repetições por bairro, ou seja, a cada um dos entrevistados por bairro; e $\mathrm{N}$ foi igual a 90 , sendo o número total de entrevistados.
A estatística T considera a possibilidade de empates de ordenação, que ocorrem freqüentemente na avaliação de respostas por meio de graus que denotam intensidade. A estatística $\mathrm{T}$ tem distribuição como o índice de $\mathrm{X}^{2}$ (Qui-quadrado), com k-1 graus de liberdade. Se o valor de $\mathrm{T}$ calculado for igual ou superior àquele tabelado, para um mesmo grau de liberdade e uma mesma probabilidade existirão diferenças significativas entre os grupos estudados.

Para as questões de números 8,11 e 12, foi utilizado o teste de hipóteses Qui-quadrado $\left(\mathrm{X}^{2}\right)$.

A estatística Qui-quadrado proporciona uma medida da discrepância entre as freqüências observadas e esperadas dos dados analisados (REGAZZI, 1997). E $\mathrm{X}^{2}$ é indicado para dados no formato binário ( 0 e 1), o que caracteriza as questões analisadas por ele.

$$
x_{c a l}^{2}=\sum_{i=1}^{k} \frac{\left[F o_{i}-F e_{i}\right]^{2}}{F e_{i}}
$$

em que:

$$
\begin{aligned}
& F o=\text { freqüência observada; e } \\
& F e=\text { freqüência esperada. }
\end{aligned}
$$

No presente trabalho, os dados foram analisados a 0,05 de probabilidade, em ambos os testes, no programa STATISTICA, versão 6.0.

As questões 1, 2, 3 e 4 foram analisadas por médias simples e desvios-padrão, visando analisar as condições socioeconômicas desses moradores, que constituem os principais motivos que os levaram à visitação e à participação em eventos ocorrentes no Parque. Já a questão 13 abriu espaço para sugestões e críticas dos moradores.

\section{RESULTADOS E DISCUSSÃO}

Os valores de probabilidade encontrados para cada questão, no nível de 5\% de significância, estão demonstrados no Quadro 3.

Observa-se, no Quadro 3, a diferença significativa entre os bairros apenas nas questões 7,8 e 9 e que as diferenças estatisticamente significantes ocorreram entre o Bairro Aglomerado da Serra e os demais. A probabilidade entre os Bairros Serra e Mangabeiras foi maior que 0,05 , portanto não havendo diferença significativa entre eles. 
Quadro 3 - Valores de probabilidade (p) encontrados em cada uma das questões. Em que: K-W = teste Kruskal-Wallis; e $\mathrm{X}^{2}=$ teste de Qui-quadrado; $\mathrm{ns}=$ não-significativo e $*=$ significativo; a $95 \%$ de probabilidade

Table 3 - Probability values found for each one of the questions. In wich: $K-W=K r u s k a l-$ Wallis test; $X^{2}=Q u i-s q u a r e d$ test; ns = non significant and $*=$ significant at $95 \%$ probability

\begin{tabular}{ccccc}
\hline Questão & Teste & $\begin{array}{c}\text { Valor de } \mathrm{p} \text { entre } \\
\text { os três bairros }\end{array}$ & Análise de $\mathrm{p}$ & $\begin{array}{c}\text { Valor de } \mathrm{p} \text { entre os } \\
\text { Bairros Serra e Mangabeiras }\end{array}$ \\
\hline 5 & $\mathrm{~K}-\mathrm{W}$ & 1 & Não-significativa & - \\
6 & $\mathrm{~K}-\mathrm{W}$ & 0,4868 & Não-significativa & - \\
7 & $\mathrm{~K}-\mathrm{W}$ & 0,004 & Significativa & 0,221 \\
8 & $\mathrm{X}^{2}$ & $<10^{-6}$ & Significativa & 0,5206 \\
9 & $\mathrm{~K}-\mathrm{W}$ & 0,0359 & Significativa & - \\
10 & $\mathrm{~K}-\mathrm{W}$ & 0,1122 & Não-significativa & - \\
11 & $\mathrm{X}^{2}$ & 0,2592 & Não-significativa & - \\
12 & $\mathrm{X}^{2}$ & 0,1712 & Não-significativa & - \\
\hline
\end{tabular}

A questão 7, que perguntava sobre a freqüência em eventos do Parque, aponta que os moradores do Aglomerado da Serra participavam muito mais dos eventos que os demais moradores do entorno. Isso pode ser explicado pelo fato de que estes possuíam menos opções de diversão e acesso à cultura que os demais, devido ao menor poder aquisitivo. Outro fator que contribuiu para essa diferença foi a existência do Projeto Meninos no Parque, que é um projeto voltado para as crianças desse bairro, o que tornava o resultado esperado. Nos Bairros Serra e Mangabeiras, 83 e $70 \%$ dos entrevistados responderam, respectivamente, que não participavam dos eventos, enquanto esea proporção caiu para $37,8 \%$ no Aglomerado da Serra. Entre os que freqüentavam, o evento mais citado foi o Fantástico Mundo da Criança, referente a 21,6\% do total de respostas.

A questão 8, que abordou os problemas devidos à proximidade entre o Parque e a residência, também apresentou divergência significativa entre o Bairro Aglomerado da Serra e os demais. Dos moradores do Serra, $80 \%$ disseram ter problemas com a proximidade do Parque com suas residências. Desses, $44 \%$ responderam ter problemas com furtos e assaltos. Entre os entrevistados do Bairro Mangabeiras, 56,7\% afirmaram ter problemas com a proximidade do Parque, sendo que 52,9\% deles também responderam ter problemas com furtos. Éimportante ressaltar que os moradores de ambos os bairros relacionaram estes crimes aos moradores do Aglomerado da Serra, sendo o PARMA, então, uma forma de acesso destes para seus bairros. Todos os entrevistados do Aglomerado da Serra responderam não haver problemas com a proximidade doPARMA; pelo contrário, eram até vantagens.

Houve, ainda, diferença significativa entre o Aglomerado da Serra e os demais bairros na questão
9, que perguntava sobre a importância do PARMA. Nos Bairros Mangabeiras e Serra, 76,7 e 50\% dos entrevistados, respectivamente, responderam que o Parque tinha valor ambiental. Dos entrevistados do Bairro Aglomerado da Serra, 56,7\% responderam que o Parque tem importância sociocultural. Um dos motivos que levam a esse resultado é o maior nível cultural dos moradores do Serra e Mangabeiras. Isso porque, como esses têm mais acesso aos meios de comunicação e à educação, podem estar mais informados sobre os movimentos ambientalistas ocorrentes atualmente no Brasil e no mundo. $\mathrm{O}$ fato de eles considerarem o Parque como uma importante área de preservação dos recursos naturais não significa que atuam de forma a conservá-la. No entanto, é justificável observar que os moradores do Aglomerado da Serra enxergavam o Parque mais como uma área de função sociocultural, pelo fato de eles não possuírem outras opções de lazer, além dessas gratuitas.

As demais questões não apresentaram divergência estatisticamente significativa entre os bairros, porém apontaram diferenças qualitativas nas respostas. Esse fato pode ser corroborado pela freqüência das visitas (questão 5), em que não houve diferenças entre o número de entrevistados dos bairros que não freqüentavam o PARMA. No entanto, os motivos dos moradores para não freqüentarem o Parque variaram de bairro para bairro, sendo que no Bairro Serra a maioria dos moradores que não freqüentavam o PARMA respondeu não ter tempo para visitá-lo ou se sentiram inseguras por causa da violência. No Bairro Aglomerado da Serra, a maioria dos moradores que não freqüentavam o PARMA disse que as portarias ficavam distantes do bairro, visto que o Parque apresentava uma portaria no Bairro Mangabeiras e duas no Bairro Serra. Os moradores que não

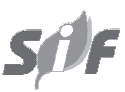

R. Árvore, Viçosa-MG, v.30, n.6, p.1033-1038, 2006 
freqüentavam o Parque do Bairro Mangabeiras disseram não ter tempo ou não possuírem condições físicas para visitá-lo devido à idade (esse foi o bairro que apresentou a maior média de idade dos moradores).

A partir das diferenças observadas, verificou-se que, para a implantação de um Plano de Educação Ambiental, será necessário atuar de forma diferente em cada bairro, respeitando a diversidade de seus moradores.

\section{CONCLUSÕES}

As diferenças observadas entre as respostas dos moradores do entorno do PARMA podem ser explicadas, principalmente, pelas diferenças socioculturais e econômicas entre essas comunidades, visto que em todas as questões contendo diferenças significativas o principal divergente foi o Bairro Aglomerado da Serra.

Entrevistados dos Bairros Serra e Mangabeiras demonstraram interesse em ajudar, de alguma forma, os vizinhos menos favorecidos do Aglomerado da Serra, desenvolvendo cursos de capacitação ou doando horas de trabalho, entre outros âmbitos, o que ajudaria a diminuir a tensão social entre os moradores dos bairros estudados. O Parque pode ser o cenário ideal para essa integração entre moradores.

As diferenças entre as afetividades do entorno do Parque com a área em questão podem ser observadas pelas questões qualitativas e quantitativas, porém todas essas diferenças levam a crer que a principal variável responsável por elas é o grande espaço que separa esses moradores, do ponto de vista socioeconômico.

\section{REFERÊNCIAS BIBLIOGRÁFICAS}

CHIARI, C.; SEEDLAND, K. Are urban green spaces optimally distributed to act as places for social integration? Results of a Geographical Information Systems (GIS) approach urban forestry research. Forest Policy and Economics, Zurich, Suíça, jul./2002. Disponível em: <http://www.elsevier.com/ locate/forpol>. Acesso em: 13 de maio de 2003.
DITT, E.H. Fragmentos florestais no Pontal do Paranapanema. São Paulo: Annablume, 2002. 140 p.

METZGER, J.P.O que é Ecologia de Paisagens? Biota Neotrópica, v.1, n.1/2, 2001. Disponível em: <http://www.biotaneotropica.org.br>. Acesso em: 27 de agosto de 2002 .

MILLER, R.W. Urban forestry: planning and managing urban greenspaces. 2. ed. New Jersey: Prentice Hall, 1997. 502 p.

MIRANDA, E.V. Plano de manejo para a Floresta Nacional de Ritápolis: versão preliminar. Viçosa, M.G.: Universidade Federal de Viçosa, 2001. 84 p.

PREFEITURA MUNICIPAL DE BELO HORIZONTE - PMBH. Informações estatísticas sobre Belo Horizonte. Estatísticas populacionais do município. Disponível em: <http://www.pbh.gov.br>.Acesso em: 19 de março de 2003a.

PREFEITURA MUNICIPAL DE BELO HORIZONTE - PMBH. Parque das Mangabeiras. Mangabeiras. Disponível em: <http:// www.pbh.gov.br/mangabeiras $>$. Acesso em: 19 de março de 2003 b.

REGAZZI, A.J. Curso de iniciação à estatística. Viçosa, MG: Universidade Federal de Viçosa, 1997. 132 p.

SAMPAIO, I.B.M. Estatística aplicada à experimentação animal. Belo Horizonte: Fundação de Ensino e Pesquisa em Veterinária e Zootecnia, 1998. $221 \mathrm{p}$. 